\title{
Superconducting and Antiferromagnetic Phases of Space-Time
}

\author{
Deepak Vaid \\ National Institute of Technology Karnataka (NITK), Surathkal, India \\ Correspondence should be addressed to Deepak Vaid; dvaid79@gmail.com \\ Received 13 October 2017; Accepted 10 December 2017; Published 31 December 2017 \\ Academic Editor: George Siopsis
}

Copyright (C) 2017 Deepak Vaid. This is an open access article distributed under the Creative Commons Attribution License, which permits unrestricted use, distribution, and reproduction in any medium, provided the original work is properly cited. The publication of this article was funded by SCOAP

A correspondence between the $S O(5)$ theory of high- $T_{C}$ superconductivity and antiferromagnetism, put forward by Zhang and collaborators, and a theory of gravity arising from symmetry breaking of a $S O(5)$ gauge field is presented. A physical correspondence between the order parameters of the unified SC/AF theory and the generators of the gravitational gauge connection is conjectured. A preliminary identification of regions of geometry, in solutions of Einstein's equations describing charged-rotating black holes embedded in de Sitter space-time, with SC and AF phases is carried out.

\section{Introduction}

Two of the outstanding problems in theoretical physics today are those of high- $T_{C}$ superconductivity (HTSC) on the one hand and quantum gravity (QG) on the other. In the case of HTSC, it has been demonstrated that the antiferromagnetic (AF) and superconducting (SC) phases $d$-wave [1-4] and $p$ wave [5] superconductors can be given a unified explanation in terms of a nonlinear sigma model for a field which behaves as a vector transformation under $S O(5)$ rotations. In QG research, it is known that general relativity with nonzero cosmological constant $(\Lambda \neq 0)$ can be obtained from a socalled BF model (a topological field theory) for a gauge field, valued in either $S O(3,2)($ for $\Lambda<0)$ or a $S O(4,1)($ for $\Lambda>0)$, by a symmetry breaking mechanism $[6,7]$. This mechanism was first outlined in a seminal paper by MacDowell and Mansouri [8] in 1977 whose motivation was to construct a unified theory of gravity and supergravity. Similar work was undertaken by Stelle and West in 1980 [9].

More recent works on these topics are the papers by Freidel and Starodubtsev [6], Randono [10, 11], and Westman and Zlosnik [12, 13]. In [14], a model similar to ours has been used to show that the early universe can undergo a spontaneous signature change from a Euclidean to Lorentzian phase. However, in contrast to the present work, the physical correspondence between phases of a real condensed matter system and various regions of a space-time has not been carried out.

The notion that geometry should have various phases is suggested by the numerical work in the field of Causal Dynamical Triangulations (CDT) [15]. Moreover in [16, 17] Ansari has demonstrated a connection between antiferromagnetism and a statistical formulation of CDT. The connection between the spin-networks used in Loop Quantum Gravity and the Ising model has recently been discussed in [18-20]. In [21], Wang has argued that the black hole horizon behaves as the surface of a topological insulator. The fact that anti-de Sitter space-time with black holes can be described as phases of a van der Waals type fluid is discussed in [22-25].

What is new in the present work, to the best of our knowledge, is that it is the first to connect the symmetry breaking on the gravitational side with a well-established model on the condensed matter side. In any situation where a symmetry is spontaneously broken, it is crucial to not only be able to identify the underlying microscopic dynamics which causes the symmetry to break and also to be able to identify and classify the various phases that result from this process. Here we are able to take the first tentative steps towards achieving both these goals.

In this work we demonstrate the equivalence between these two theoretical frameworks. The picture resulting from our line of reasoning is that of a space-time with nonzero 
$\Lambda$, as described by classical general relativity, emerging via symmetry breaking of a topological quantum field theory (TQFT), defined on a four-dimensional manifold. The superconducting phase can be identified with the near horizon geometry of a charged black hole and the antiferromagnetic phase can be identified with the geometry far from the horizon of a rotating black hole. A mixed phase, consisting of both SC and AF phases, would correspond to the space-time of a charged, rotating black hole.

The plan of the paper is as follows. In Section 2 we explain why the theory of HTSC should have effective description in terms of a $S O(5)$ gauge theory. In Sections 2.1 and 2.2, we define the order parameters and symmetry generators of the microscopic theory underlying HTSC and explain how the $S O(5)$ symmetry arises from these structures. In Sections 3 and 4 we outline the basic structure of Einstein-Cartan gravity and how it can be seen as arising from symmetry breaking of a TQFT represented by a BF theory.

\section{2. $\mathrm{SO}(5)$ Model of High Temperature Superconductivity}

One can ask why the group $S O(5)$ should have anything to do with the description of the SC or AF phases (the phase diagram for HTSC is shown in Figure 1) in condensed matter systems and, for that matter, why do we need a unified description of the two phases in the first place. There are three reasons [4] to believe that this should be the case:

(1) In 1988, Chakravarty et al. [27] demonstrated that the nonlinear sigma model for a field with $S O(3)$ symmetry gives a good description of the properties of a twodimensional $(2+1)$ Heisenberg antiferromagnet in the low-temperature, long-wavelength regime.

(2) The behavior of the superconducting state is known to be well-described by a so-called " $X Y$ " model for a $U(1)$ gauge field.

(3) Both $d$-wave SC and AF can be described in terms of the behavior of singlet pairs in the Hubbard model at half-filling. These singlet pairs can describe either an AF phase, a SC phase, or a so-called "spin-bag" phase where both the phases coexist.

Now, if both AF and SC arise in different regimes of a system with the same underlying physics, the Hubbard model at half-filling, and can coexist under certain conditions, it follows that one would be well-advised to seek out a lowtemperature, long-wavelength effective field theory which can describe both phases. Such a theory should contain a $S O(3) \times U(1)$ symmetry, which should arise after some symmetry breaking transition. The smallest gauge group that can accommodate such a symmetry among its subgroups is $\mathrm{SO}(5)$.

In [1-5] it was shown that a nonlinear sigma model for a field with an $S O(5)$ gauge symmetry can describe the physics of both the AF and SC phases. The ten independent elements of the $S O(5)$ Lie algebra can be identified with (see $(6 a)-(6 c))$ the three components of the generator of total spin

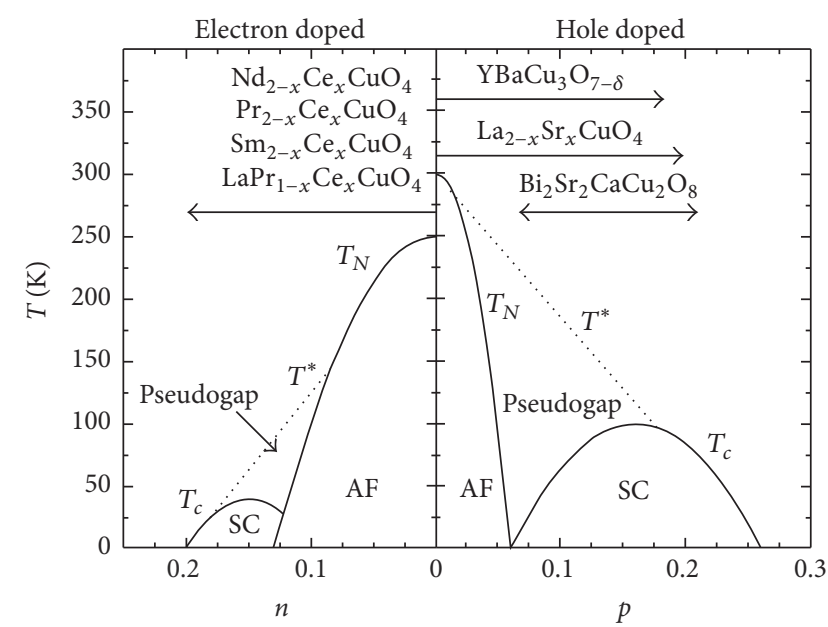

FIGURE 1: Simplified doping-dependent phase diagram of cuprate superconductors for both electron $(n)$ and hole $(p)$ doping. The phases shown are the antiferromagnetic (AF) phase close to zero doping, the superconducting phase around optimal doping, and the pseudogap phase. Doping ranges possible for some common compounds are also shown, after [26].

$\vec{S}$, the total charge operator $Q$, and the six real and imaginary components of the $\vec{\pi}$ operator (which "rotates" the AF phase into the SC phases and vice versa).

As argued by Zhang [4], the physical picture of the transition between the AF and SC states is the following. The overall system is described by some microscopic Hamiltonian describing the interaction between electrons on a lattice. Below some characteristic temperature $T_{M F}$, and below some critical value of an external parameter $\lambda$ (typically this is the electron or hole doping in the cuprate superconductors though it can also be the pressure or some other external parameter) electrons on neighboring sites tend to from singlet bound pairs or dimers. The AF and the SC phase are different states in which this dimer collective can form. When the dimers are not free to move, due to the lack of vacancies on the lattice, the collective forms a dimer "solid" which corresponds to the AF phase (left side of Figure 2). As $\lambda$ is varied, the dimer solid begins to melt and forms a fluid which corresponds to the SC phase (right side of Figure 2). At the transition between the two phases one will have regions where both the solid and liquid phases are present. This corresponds to the "spin-bag" phase where both AF and SC coexist.

2.1. Order Parameters. We will define our creation and annihilation operators in the spatial representation in order to make better contact with the physical system. The interested reader can find the momentum space expressions of these operators in Section 2.2.

Let us introduce some notation. In order to study antiferromagnetic ordering we need at least two neighboring sites with spins $\vec{S}_{1}$ and $\vec{S}_{2}$. Let $\vec{S}=\vec{S}_{1}+\vec{S}_{2}$ be the total spin on the two sites and $\vec{N}=\vec{S}_{1}-\vec{S}_{2}$ be the Néel vector which is measures AF order. Let $\left\{c_{\alpha}^{\dagger}, c_{\alpha}\right\}$ and $\left\{d_{\alpha}^{\dagger}, d_{\alpha}\right\}$ be the 


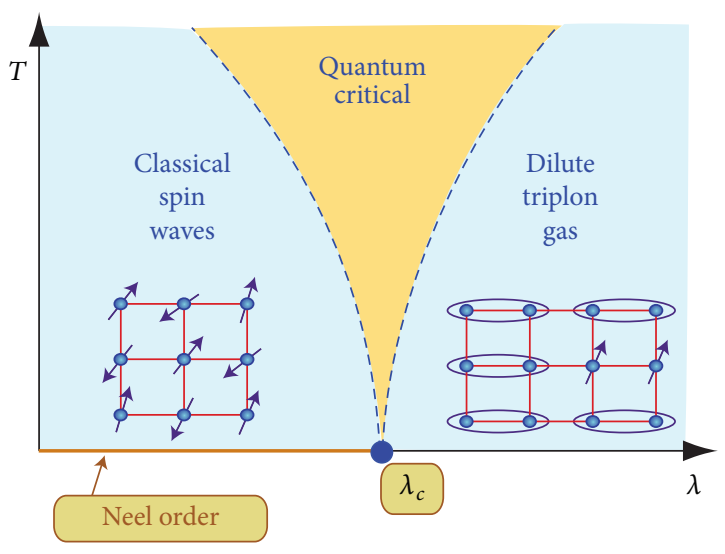

FIGURE 2: Schematic illustration of the SC and AF phases of HTSC. For $\lambda<\lambda_{c}$, the system favors AF alignment of neighboring spins. For $\lambda>\lambda_{c}$ SC emerges. The quasiparticles in the AF phase spin waves and in the SC phase they are "triplons," dimers consisting of two neighboring spins which are aligned parallel to each other. In between the AF and SC phases there is a pseudogap phase which consists of a mixture of SC and AF phases.

fermion creation and annihilation operators (with $\alpha=1,2$ the spinorial index) on sites 1 and 2 , respectively. $\sigma^{i}, i=$ $x, y, z$ are the Pauli spin-matrices. In terms of these operators the AF order parameter can be defined as follows [2]:

$$
\begin{aligned}
N^{i} & =\frac{1}{2} \sum_{\alpha^{\prime}, \alpha=1,2}\left(c_{\alpha^{\prime}}^{\dagger} \sigma_{\alpha^{\prime} \alpha}^{i} c_{\alpha}-d_{\alpha^{\prime}}^{\dagger} \sigma_{\alpha^{\prime} \alpha}^{i} d_{\alpha}\right) \\
& =\frac{1}{2}\left(c^{\dagger} \sigma^{i} c-d^{\dagger} \sigma^{i} d\right),
\end{aligned}
$$

where in the first line we have explicitly indicated the sum over the spinorial indices, which are suppressed for simplicity in the second line. Henceforth the spinorial indices will be shown explicitly only if needed.

The SC order parameter is defined as the operator which measures the formation of singlet pairs of equal and opposite spins on neighboring sites. To do so first we first define the operator $\Delta$ :

$$
\Delta^{\dagger}=\frac{-i}{2} c^{\dagger} \sigma^{y} d^{\dagger}
$$

Working in the spin basis, where $\sigma^{y}$ is diagonal, the above expression can also be written as follows:

$$
\Delta^{\dagger}=\frac{-i}{2}\left(\begin{array}{cc}
c_{\uparrow}^{\dagger} & c_{\downarrow}^{\dagger}
\end{array}\right)\left(\begin{array}{cc}
0 & -i \\
i & 0
\end{array}\right)\left(\begin{array}{l}
d_{\uparrow}^{\dagger} \\
d_{\downarrow}^{\uparrow}
\end{array}\right)=\frac{1}{2}\left(-c_{\uparrow}^{\dagger} d_{\downarrow}^{\dagger}+c_{\downarrow}^{\dagger} d_{\uparrow}^{\dagger}\right) .
$$

In terms of $\Delta$, the two SC order parameters are as follows:

$$
\begin{aligned}
& n_{1}=\frac{\Delta^{\dagger}+\Delta}{2} ; \\
& n_{5}=\frac{\Delta^{\dagger}-\Delta}{2 i} .
\end{aligned}
$$

Setting $n_{2}=N^{x}, n_{3}=N^{y}$ and $n_{4}=N^{z}$, we can finally define the five-dimensional "superspin" vector:

$$
n_{a}=\left(n_{1}, n_{2}, n_{3}, n_{4}, n_{5}\right) \text {. }
$$

2.2. Symmetry Generators. In order to able to write down the generators of the $S O(5)$ Lie algebra, with respect to which $n_{a}$ transforms as an $S O(5)$ vector, we need to define the operators which generate the symmetries of the system. These are $S_{i}, Q$, and $\pi_{i}$ which correspond, respectively, to the total spin, total charge, and AF-to-SC transformation operators, respectively. They can be defined as follows:

$$
\begin{aligned}
S_{i} & =\frac{1}{2}\left(c^{\dagger} \sigma^{i} c+d^{\dagger} \sigma^{i} d\right) \\
Q & =\frac{1}{2}\left(c^{\dagger} c+d^{\dagger} d-2\right) \\
\pi_{i}^{\dagger} & =-\frac{1}{2} c^{\dagger} \sigma^{i} \sigma^{y} d^{\dagger} ; \\
\pi & =\left(\pi^{\dagger}\right)^{\dagger} .
\end{aligned}
$$

The operator $\pi=\left(\pi_{x}, \pi_{y}, \pi_{z}\right)$ rotates the AF order parameter into the SC order parameter and vice versa [4]. This can be seen by calculating the commutation relations between $\pi$ and the AF and SC order parameters $N_{i}, \Delta$ :

$$
\begin{aligned}
{\left[\pi_{i}^{\dagger}, N_{j}\right] } & =i \delta_{i j} \Delta^{\dagger} ; \\
{\left[\pi_{i}^{\dagger}, \Delta\right] } & =i N_{i} .
\end{aligned}
$$

The operators $S_{i}, Q$ and $\pi_{i}$ can be arranged in the form of a $5 \times 5$ matrix $L_{a b}$ as in the following:

$$
\begin{aligned}
& L_{a b}
\end{aligned}
$$

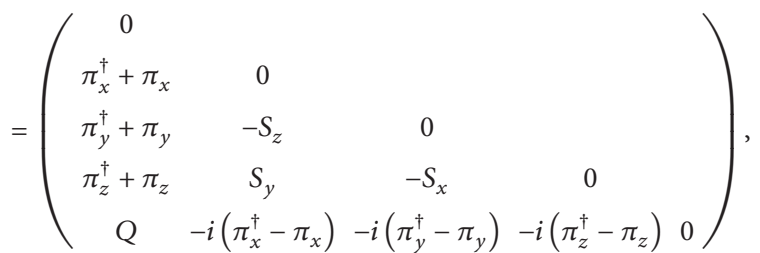

where $L_{a b}=-L_{b a}$. The elements of this matrix satisfy the commutation relations:

$$
\left[L_{a b}, L_{c d}\right]=i\left(\delta_{a c} L_{b d}-\delta_{a d} L_{b c}-\delta_{b c} L_{a d}+\delta_{a d} L_{b c}\right),
$$

which are the commutation rules satisfied by the generators of the Lie algebra of the group $S O(5) . L_{a b}$ and $n_{a}$ satisfy the following:

$$
\left[L_{a b}, n_{c}\right]=-i\left(\delta_{a c} n_{b}-\delta_{b c} n_{a}\right)
$$

which shows that $n_{a}$ transforms as the vector representation of the group $S O(5)$ generated by $L_{a b} . L_{a b}$ and $n_{c}$ can also be seen to be conjugate variables [2], just as the momentum and position $p, q$ are in the ordinary harmonic oscillator. Thus in 
terms of these objects we can write down the Hamiltonian for $S O(5)$ effective theory of AF and SC:

$$
\begin{aligned}
H_{S O 5}= & \frac{1}{2 \chi} \sum_{x, a<b} L_{a b}^{2}(x)+\frac{\rho}{2} \sum_{\left\langle x, x^{\prime}\right\rangle, a} n^{i}(x) n_{i}\left(x^{\prime}\right) \\
& +\sum_{x, a<b} B_{a b}(x) L_{a b}(x)+\sum_{x} V(n(x)),
\end{aligned}
$$

where the various terms correspond to, respectively, the kinetic energy of $S O(5)$ rotors $\left(\sim L^{2}\right)$, the coupling between rotors on different sites $\left(\sim n^{2},\langle\cdots\rangle\right.$ denotes sum over nearest neighbors), coupling between an external field and the momenta of the rotors $(\sim B L)$, and a symmetry breaking term $(V(n))$ which breaks the $S O(5)$ symmetry down to $S O(3) \times$ $U(1)$.

\section{Cartan Decomposition}

We now come to the gravity side of the picture. Our ingredients are a four-dimensional manifold $\mathscr{M}^{4}$ on which we have a $S O(4,1)$ or $S O(3,2)$ connection $A_{\mu}^{I}$, depending on whether $\Lambda>0$ and $\Lambda<0$, respectively. There is no metric structure on this manifold to begin with. This connection can then be decomposed into two parts [7]:

$$
A=\omega+\frac{\epsilon}{l} e,
$$

where $\omega_{\mu}^{I}$ is identified with an $\mathfrak{g} \mathfrak{p}(3,1)$ connection and $e_{\mu}^{I}$ is a four-dimensional frame field. $\epsilon=+1$ when $\Lambda>0$ and $\epsilon=-1$ when $\Lambda<0$. The curvature $F[A]$ of the connection can then be written as follows:

$$
F[A]_{J}^{I}=\mathbf{d} A_{J}^{I}+A_{K}^{I} \wedge A_{J}^{K},
$$

where $I, J, K=0,1,2,3$, 4 label the elements of the $\mathfrak{g} \mathfrak{v}(4,1)$ $(\mathfrak{g} \mathfrak{o}(3,2)$, resp. $)$ matrices, and the space-time indices are suppressed. $\mathbf{d}$ is the exterior derivative. In terms of these indices, (12) can be written as follows:

$$
\begin{aligned}
& A_{b}^{a}=\omega_{b}^{a} ; \\
& A^{a}{ }_{4}=\frac{1}{l} e^{a} ; \\
& A_{a}^{4}=-\frac{\epsilon}{l} e_{a},
\end{aligned}
$$

where $a, b=0,1,2,3$. This can be more clearly seen in the explicit matrix form:

$$
A_{J}^{I}=\left(\begin{array}{ccccc}
0 & \omega_{1}^{0} & \omega_{2}^{0} & \omega_{3}^{0} & \frac{e^{0}}{l} \\
\omega_{0}^{1} & 0 & \omega_{2}^{1} & \omega_{3}^{1} & \frac{e^{1}}{l} \\
\omega_{0}^{2} & \omega_{1}^{2} & 0 & \omega_{3}^{2} & \frac{e^{2}}{l} \\
\omega_{0}^{3} & \omega_{1}^{3} & \omega_{2}^{3} & 0 & \frac{e^{3}}{l} \\
\frac{\epsilon e^{0}}{l} & -\frac{\epsilon e^{1}}{l} & -\frac{\epsilon e^{2}}{l} & -\frac{\epsilon e^{3}}{l} & 0
\end{array}\right) .
$$

$\omega^{0}{ }_{i}(i=1,2,3)$ are the generators of boosts, $\omega^{i}{ }_{j}(i, j=1,2,3$; $i \neq j)$ are the generators of spatial rotations $\left(\omega^{0}{ }_{1}=-\omega^{1}{ }_{0}\right.$ and $\left.\omega_{2}^{1}=-\omega^{2}{ }_{1}\right)$, and $e^{a} / l(a=0,1,2,3)$ are the generators of translations. The gauge curvature can then be expanded as follows. For the $\mathfrak{s} \mathfrak{v}(3,1)$ part,

$$
\begin{aligned}
F_{b}^{a} & =\mathbf{d} A^{a}{ }_{b}+A^{a}{ }_{c} \wedge A^{c}{ }_{d}+A^{a}{ }_{4} \wedge A^{4}{ }_{b} \\
& =\mathbf{d} \omega^{a}{ }_{b}+\omega^{a}{ }_{c} \wedge \omega^{c}{ }_{d}-\frac{\epsilon}{l^{2}} e^{a} \wedge e_{b} \\
& =R^{a}{ }_{b}-\frac{\epsilon}{l^{2}} e^{a} \wedge e_{b} .
\end{aligned}
$$

Similarly the $\mathbb{R}(3,1)$ part of the curvature is given by the following:

$$
\begin{aligned}
F^{a}{ }_{4} & =\mathbf{d} A^{a}{ }_{4}+A^{a}{ }_{b} \wedge A^{b}{ }_{4}=\frac{1}{l}\left(\mathbf{d} e^{a}+\omega^{a}{ }_{b} \wedge e^{b}\right) \\
& =\frac{1}{l} \mathbf{D}_{\omega} e^{a},
\end{aligned}
$$

where $\mathbf{D}_{\omega}$ is the antisymmetrized covariant derivative operator with respect to the connection $\omega$. Finally we see that the various components of the gauge field strength can be written as follows:

$$
\begin{aligned}
& F_{b}^{a}=R_{b}^{a}-\frac{\epsilon}{l^{2}} e^{a} \wedge e_{b} \\
& F_{4}^{a}=\frac{1}{l} \mathbf{D}_{\omega} e
\end{aligned}
$$

where $R$ is the curvature of a $\mathfrak{g} \mathfrak{o}(3,1)$ connection $\omega$ and $e$ is a $\mathfrak{S} \mathfrak{o}(3,1)$ valued one-form.

\section{4. $B F$ Theory}

The action for a topological theory on a manifold $\mathscr{U}$ with local gauge group $G$ is given by:

$$
S_{B F}=\int\left(B_{I J} \wedge F^{I J}\right)
$$

where $B$ and $F$ are a ( $n-2)$-form and a 2 -form respectively on $\mathscr{M}$ and which takes values in the Lie algebra $\mathfrak{g}$ of $G . F$ is the field strength for a connection $A$. The configuration variables are the gauge connection $A$ and the two-form field $B$ and the action is invariant under $S O(5)$ transformations of the gauge field. Varying the action with respect to these variables we find the two equations of motion [6]:

$$
\begin{aligned}
\frac{\delta S_{B F}}{\delta B} & =0 \Longrightarrow \\
F_{I J} & =0 \\
\frac{\delta S_{B F}}{\delta A} & =0 \Longrightarrow \\
\mathbf{D}_{A} B^{I J} & =0,
\end{aligned}
$$


where $F_{I J}=\mathbf{d} A_{I J}+A_{I}{ }^{K} \wedge A_{K J}$ is the curvature tensor and $\mathbf{D}_{A}$ is the covariant derivative with respect to the gauge connection $A$. We have made use of the fact that

$$
\delta F_{I J}=\mathbf{d}\left(\delta A_{I J}\right)+A_{I}{ }^{K} \wedge \delta A_{K J}=\mathbf{D}_{A}\left(\delta A_{I J}\right)
$$

followed by a partial integration in order to obtain (20a) and (20b).

Since the field strength is identically zero everywhere, in the present form, this action describes a system with no local degrees of freedom. The value of $S_{B F}$ when evaluated on a given manifold, for any choice of $B$ and $A$, will only yield information about the topology of the manifold. Thus (19) is the action for a topological field theory or TFT and as such has no correspondence with classical general relativity. The situation changes, however, when we add a term to the action quadratic in the $B$ field which corresponds to the breaking of the $S O(5)$ symmetry of the theory resulting in a theory with propagating local degrees of freedom. The modified action is as follows [6]:

$$
S_{B F}^{\prime}=\int\left(B_{I J} \wedge F^{I J}-\frac{1}{2} B^{I J} \wedge B^{K L} \epsilon_{I J K L M} v^{M}\right),
$$

where $v^{M}$ is a fixed $S O(5)$ vector pointing in a preferred direction. It is this choice of a preferred direction that breaks the $S O(5)$ symmetry, in much the same way as the choice of a preferred direction for spins breaks the symmetry of the Ising model and allows the ferromagnetic phase to appear from an initially disordered phase where the spins point in arbitrary directions. follows:

The equations of motion for the modified action are as

$$
\begin{aligned}
\frac{\delta S_{B F}^{\prime}}{\delta B} & =0 \Longrightarrow \\
F_{I J} & =\frac{1}{2} \epsilon_{I J K L M} B^{K L} v^{M} \\
\frac{\delta S_{B F}^{\prime}}{\delta A} & =0 \Longrightarrow \\
\mathbf{D}_{A} B^{I J} & =0 .
\end{aligned}
$$

Now we can always choose our coordinates in the $S O(5)$ space such that $v^{M}$ has only one nonvanishing component such that $v^{M}:=(0,0,0,0, \alpha / 2)$. Then the equation of motion for the $B$ field in (23a) and (23b) becomes

$$
\begin{aligned}
& F_{a b}=\frac{\alpha}{4} \epsilon_{a b c d} B^{c d} \\
& F_{a 4}=0
\end{aligned}
$$

where $a, b \in\{0,1,2,3\}$, whereas the e.o.m for the gauge connection is unchanged. The second of these equations in combination with (18b) tells us that

$$
\mathbf{D}_{\omega} e=0
$$

that is, the torsion of the gauge connection is zero. Contracting both sides of (24a) with $\epsilon_{a b}^{e f}$ we obtain

$$
B^{e f}=\frac{1}{\alpha} \star F^{e f},
$$

where $\star$ is the Hodge dual operator (contraction with $\epsilon_{a b c d}$ ). Substituting the solution for $B(26)$ into the modified action and using the fact that $F_{a 4}=0(24 \mathrm{~b})$, we find

$$
\begin{aligned}
S_{B F}^{\prime}= & \int\left(-\frac{1}{\alpha} \star F_{a b} \wedge F^{a b}-\frac{1}{\alpha} \star F^{a b} \wedge F_{a b}\right) \\
= & -\frac{1}{2 \alpha} \int F^{a b} \wedge \star F_{a b} \\
= & -\frac{1}{2 \alpha} \int\left(R^{a b}-\frac{\epsilon}{l^{2}} e^{a} \wedge e^{b}\right) \\
& \wedge \star\left(R_{a b}-\frac{\epsilon}{l^{2}} e_{a} \wedge e_{b}\right),
\end{aligned}
$$

where in the third line we have utilized the identity (18a). Finally we have

$$
\begin{aligned}
S_{B F}^{\prime} & =-\frac{1}{2 \alpha} \int\left[R^{a b} \wedge \star R_{a b}+\frac{\epsilon^{2}}{l^{4}} e^{a} \wedge e^{b} \wedge \star\left(e_{a} \wedge e_{b}\right)\right. \\
& \left.-\frac{2 \epsilon}{l^{2}} R^{a b} \wedge \star\left(e_{a} \wedge e_{b}\right)\right] .
\end{aligned}
$$

The last two terms give us the Palatini action (The connection formalism and the first-order Palatini action for general relativity are reviewed in a forthcoming review article on LQG [28].) for general relativity with a cosmological constant, while the first term is a topological term whose variation vanishes due to the Bianchi identity.

\section{Physical Interpretation}

It is straightforward to see the correspondence between the operators for charge, rotations, and translations (acting on the electron wave function which) forms the components of the $S O(5)$ connection (B.4) and the operators defined in the space-time connection given in (15). First let us write down the form of the $5 \times 5$ matrix generators of the Lie algebras of $\mathfrak{S} \mathfrak{o}(4,1), \mathfrak{i} \mathfrak{g} \mathfrak{o}(3,1)$, and $\mathfrak{S} \mathfrak{o}(3,2)$ in the following suggestive form $[7$, p. 10]:

$$
\begin{gathered}
\left(\begin{array}{ccccc}
0 & b^{1} & b^{2} & b^{3} & \frac{p^{0}}{l} \\
b^{1} & 0 & j^{3} & -j^{2} & \frac{p^{1}}{l} \\
b^{2} & -j^{3} & 0 & j^{1} & \frac{p^{2}}{l} \\
b^{3} & j^{2} & -j^{1} & 0 & \frac{p^{3}}{l} \\
\frac{\epsilon p^{0}}{l} & -\frac{\epsilon p^{1}}{l} & -\frac{\epsilon p^{2}}{l} & -\frac{\epsilon p^{3}}{l} & 0
\end{array}\right) \\
=j^{i} J_{i}+b^{i} B_{i}+\frac{1}{l} p^{a} P_{a},
\end{gathered}
$$


TABLE 1: Physical correspondence between operators in the condensed matter system and in the gravitational theory.

\begin{tabular}{lcc}
\hline & $L_{I J}$ & $A_{I J}$ \\
\hline \multirow{3}{*}{ Rotations } & $S_{x}$ & $-\omega^{3}{ }_{2}$ \\
& $S_{y}$ & $\omega^{3}{ }_{1}$ \\
& $S_{z}$ & $-\omega^{2}{ }_{1}$ \\
\hline \multirow{3}{*}{ Boosts } & $\pi_{x}^{\dagger}+\pi_{x}$ & $\omega^{1}{ }_{0}$ \\
& $\pi_{y}^{\dagger}+\pi_{y}$ & $\omega^{2}{ }_{0}$ \\
& $\pi_{z}^{\dagger}+\pi_{z}$ & $\omega^{3}{ }_{0}$ \\
\hline \multirow{3}{*}{ Translations } & $i\left(\pi_{x}^{\dagger}+\pi_{x}\right)$ & $\epsilon e^{1} / l$ \\
& $i\left(\pi_{y}^{\dagger}+\pi_{y}\right)$ & $\epsilon e^{2} / l$ \\
& $i\left(\pi_{z}^{\dagger}+\pi_{z}\right)$ & $\epsilon e^{3} / l$ \\
\hline Charge & $Q$ & $\epsilon e^{0} / l$ \\
\hline
\end{tabular}

where $J_{i}$ are the generators of rotations, $B_{i}$ generate boosts, and $P_{a}=\left(P_{0}, P_{i}\right)$ generate translations. The value of the factor $\epsilon$ determines the type of the algebra. If $\epsilon$ is $-1,0$, or 1 , the Lie algebra the above matrix describes $\mathfrak{\mathfrak { o }}(4,1)$, $\mathfrak{i} \mathfrak{\mathfrak { o }}(3,1)$, or $\mathfrak{S o}(3,2)$ respectively.

Table 1 illustrates this correspondence.

\section{Discussion: Phases of Space-Time}

On the condensed matter side, it is understood that the $S O(5)$ formalism for high- $T_{C}$ superconductivity and antiferromagnetism is only an approximation (or effective field theory) $[29,30]$ that arises in the long-wavelength low-energy limit of the physics of some underlying fundamental dynamics. In [2, 31] several examples of microscopic Hamiltonians are given whose long-wavelength theory explicitly exhibit the $S O(5)$ symmetry. A recurring example of an exact microscopic Hamiltonian in the case of high- $T_{C} \mathrm{SC} / \mathrm{AF}$ is that of the tightbinding Hubbard model. In $[32,33]$ we pointed out that the behavior of black hole entropy in LQG suggests a connection between the physics of a black hole horizon and that of the quantum hall effect. There we suggested the Hubbard model as a candidate microscopic Hamiltonian for describing the physics of a black hole horizon. The present work provides support for this proposal. This addresses the question of the microscopic origin of the effective $S O(5)$ theory in the gravitational context.

Knowledge of the detailed phase diagram of HTSC/AF also allows us to make concrete suggestions regarding the possible phases which space-time geometry can manifest. The important aspect is the ability to identify the various phases, superconducting, antiferromagnetic, ferromagnetic, spin-bag, and so forth, with the various solutions of Einstein's equations. To do so we can refer to the dictionary given in Table 1.

It is important at this stage to point out a crucial difference between Zhang's SO(5) system and our model. In Zhang's model the $S O(5)$ symmetry is a global symmetry: the Lie algebra generators in (B.4) do not have any spatial dependence. In our model we have gauged this symmetry and made it local: Lie algebra generators in (15) have a spacetime index (which was not shown in the text to avoid clutter).
For instance, the rotation generators $\omega^{i}{ }_{j}$ are more accurately written as $\omega_{\mu}{ }^{i}{ }_{j}$ with a space-time index $\mu$.

In the AF phase the symmetry generator is given by the spin vector $\mathbf{S}=\left(S_{x}, S_{y}, S_{z}\right)$ (6a). The dictionary in Table 1 tells us that on the gravitational side this corresponds to the components of the $\mathfrak{s} \mathfrak{v}(5)$ connection which correspond to spatial rotations $\left(-\omega^{3}{ }_{2}, \omega^{3}{ }_{1},-\omega^{2}{ }_{1}\right)$ in the symmetry broken theory. Thus in order to associate a geometric configuration with an AF phase, we should look for a solution of Einstein's equations where rotations in the spatial planes are determined. An example is space-time of a Kerr-de Sitter (The theory we are considering has $\Lambda \neq 0$; thus one has to work with the de Sitter/anti-de Sitter generalization of the Kerr space-time.) black hole, which describes a rotating black hole. Observers outside a Kerr-de Sitter black hole will experience a space-time with broken rotational invariance, with the rotation axis of the black hole defining a preferred direction in space, and far from the horizon, the generators of spatial rotations $\left(-\omega^{3}{ }_{2}, \omega^{3}{ }_{1},-\omega^{2}{ }_{1}\right)$ will reach a constant, nonzero value. Thus, the geometry experienced by observers far from the horizon of a rotating black hole can be identified with the antiferromagnetic phase.

For the SC phase it is, at present, not clear to us as to what geometric configuration should be identified with it. A guess would be that the geometry near or inside the horizon of a charged, Reissner-Nordstrom, black hole can be identified with a SC phase (It is known that a scalar field living in the charged black hole background will undergo symmetry breaking leading to formation of a superconducting condensate in the near horizon region [34-37]. This observation would appear to buttress our identification of a charged black hole geometry with the SC phase of a symmetry broken $S O(5)$ theory.). If we consider the case of black hole which is both rotating and charged Kerr-Newman (once again with the caveat that the black hole is embedded in a bulk de Sitter space-time), then it would appear that the AF phase can be identified with the bulk geometry far from the horizon and the SC phase with the bulk geometry in the interior of the black hole. Of course, this identification is, as yet, qualitative and requires detailed analytical investigation before it can be fully accepted. However, this does tells us the general direction one must follow for identifying phases of geometry with the phases encountered in condensed matter.

6.1. From $S O(5)$ to $S O(3,2)$ or $S O(4,1)$. In much of the literature it is commonly assumed that one can work with the Euclidean signature space-time and eventually Wick rotate one of the dimensions to yield a Lorentzian spacetime. The role of time in the Lorentzian case is played by the "temperature," in the Euclidean picture. For instance in order to calculate the temperature of Hawking radiation emitted from a black hole a common technique that is used [38, Section 3.2.2] is to analytically continue the black hole metric to Euclidean signature (whence the "time" becomes an imaginary quantity $t_{E}=i t$ ) and to identify the periodicity in the imaginary component of the Euclidean time with the inverse temperature $\beta=1 / k_{B} T \sim \mathfrak{J}\left(t_{E}\right)$. 
TABLE 2

\begin{tabular}{lcc}
\hline Quantity & Symbols & Range \\
\hline 5D Clifford algebra & $I, J, K, \ldots$ & $\{0,1,2,3,4\}$ \\
Generators of $S O(5)$ & $I J, J K, \ldots$ & \\
Generators of $S O(4,1)$ and $S O(3,2)$ & $a, b, c, \ldots$ & $\{0,1,2,3\}$ \\
Generators of $S O(3)$ and $S U(2)$ & $i, j, k, \ldots$ & $\{1,2,3\}$ \\
Space-time coordinates & $\mu, \nu, \alpha, \beta, \ldots$ & $\{0,1,2,3\}$ \\
\hline
\end{tabular}

It is true that, in this work, we have been somewhat careless in identifying the $S O(5)$ gauge group of the condensed matter theory with the $S O(4,1)$ or $S O(3,2)$ gauge groups of the gravity theory; however, we are confident that a more detailed investigation will bear out the correctness of the essential aspects of this approach.

\section{Appendix}

\section{A. Notational Conventions}

For the reader's convenience let us clarify some aspects of the notation used in this paper as shown in Table 2.

For the most part, space-time indices $\mu, \nu, \ldots$ are suppressed. One-forms correspond to objects with one spacetime index: $V_{\mu}$. Two-forms are objects with two space-time indices $F_{\mu \nu}$, which are antisymmetric in those indices, that is, $F_{\{\mu \nu\}}=0$, where $\{\cdots\}$ denotes symmetrization over the enclosed indices.

The tetrad $e_{\mu}{ }^{I}$ can be thought of as a space-time field (labeled by $\mu$ ) which, at each point of our space-time manifold, gives us a vector (labeled by $I$ ), or more precisely an element of the five-dimensional Clifford algebra, which rotates under the respective gauge transformations.

The "wedge" product between one-forms and two-forms is defined as the completely antisymmetric outer product between two given objects. For instance, given a one-form $e_{\mu}$ and a two-form $F_{\mu \nu}$, the wedge product between the two would give a three-index object completely antisymmetric in all the indices:

$$
e \wedge F \equiv \epsilon^{\mu \nu \delta} e_{\mu} F_{\nu \delta}
$$

The action for $B F$ theory written with space-time indices shown explicitly is as follows:

$$
S_{B F}=\int d^{4} x B^{I J} \wedge F_{I J} \equiv \int d^{4} x \epsilon^{\mu \nu \alpha \beta} B_{\mu \nu}{ }^{I J} F_{\alpha \beta I J} \cdot
$$

\section{B. Momentum Space Representation}

$c_{\mathbf{p}, \uparrow \downarrow}^{\dagger}\left(c_{\mathbf{p}, \uparrow \downarrow}\right)$ is the operator which creates (destroys, resp.) an electron with momentum $\mathbf{p}$ and given spin $(\uparrow$ or $\downarrow$ ). With these in hand we can define the operators for spin $(S)$, momenta $(\pi)$, and total charge $(Q)$ for electrons near the Fermi surface. These are as follows:

$$
\begin{aligned}
S_{i} & =\sum_{\mathbf{k}, \alpha, \beta} c_{\mathbf{k} \alpha}^{\dagger} \sigma_{\alpha \beta}^{i} \mathcal{c}_{\mathbf{k}, \beta} \\
\pi_{i}(\mathbf{k}) & =\frac{1}{4} \sum_{\mathbf{p}, \alpha, \beta} g(\mathbf{k}, \mathbf{p}) c_{-\mathbf{p}, \alpha}\left(\sigma^{y} \sigma^{i}\right)_{\alpha \beta} c_{\mathbf{p}, \beta} \\
Q & =\frac{1}{2} \sum_{\mathbf{k}, \alpha}\left(c_{\mathbf{k}, \alpha}^{\dagger} c_{\mathbf{k}, \alpha}-\frac{1}{2}\right) .
\end{aligned}
$$

These are the symmetry generators of the system. Here $g(\mathbf{k}, \mathbf{p}) \equiv g(\mathbf{k}-\mathbf{p})$ is a function of the electron momenta in terms of which the operator for the superconducting gap $\Delta$ can be written as follows $[3,4]$ :

$$
\Delta^{\dagger}=\frac{1}{2} \sum_{\mathbf{k}} g(\mathbf{k}) c_{\mathbf{k} \uparrow}^{\dagger} c_{-\mathbf{k} \downarrow}^{\dagger}
$$

Thus $g(\mathbf{k})$ possesses the symmetries of the gap function $\Delta_{\mathbf{k}}$. For the case of $d$-wave HTSC, it has the following form:

$$
g(\mathbf{k})=\cos k_{x}-\cos k_{y} .
$$

These operators can be arranged in the form of a $5 \times 5$ matrix $L_{I J}$ as in

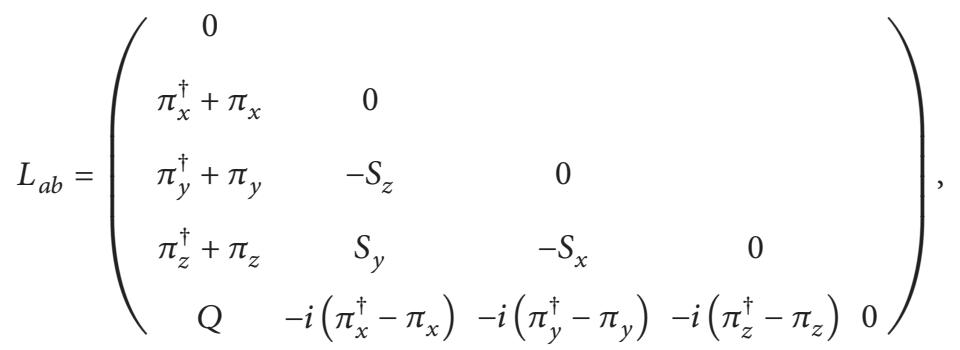

where $L_{a b}=-L_{b a}$. It can be shown that, if $|g(\mathbf{k})|^{2}=1$, the elements of this matrix satisfy the commutation relations:

$$
\left[L_{a b}, L_{c d}\right]=i\left(\delta_{a c} L_{b d}-\delta_{a d} L_{b c}-\delta_{b c} L_{a d}+\delta_{a d} L_{b c}\right),
$$

which are the commutation rules satisfied by the generators of the Lie algebra of the group $S O(5)$. Zhang $[3,4]$ suggests that the behavior of high- $T_{C}$ superconductors can be characterized by introducing a five-dimensional superspin vector 
$n^{I}$, whose components can be identified with the various AF and SC order parameters as follows:

$$
\begin{aligned}
& n^{1}=\Delta^{\dagger}+\Delta \\
& n^{5}=-i\left(\Delta^{\dagger}-\Delta\right) \\
& n^{2}=\sum_{\mathbf{k}, \alpha, \beta} c_{\mathbf{k}+\Pi, \alpha}^{\dagger} \sigma_{\alpha \beta}^{x} \mathcal{c}_{\mathbf{k}, \beta} \\
& n^{3}=\sum_{\mathbf{k}, \alpha, \beta} c_{\mathbf{k}+\Pi, \alpha}^{\dagger} \sigma_{\alpha \beta}^{y} \mathcal{c}_{\mathbf{k}, \beta} \\
& n^{4}=\sum_{\mathbf{k}, \alpha, \beta} \mathcal{c}_{\mathbf{k}+\Pi, \alpha}^{\dagger} \sigma_{\alpha \beta}^{z} \mathcal{c}_{\mathbf{k}, \beta},
\end{aligned}
$$

where $\Pi=(\pi, \pi)=\left(k_{x}, k_{y}\right)$ refers to the electron momenta in the two-dimensional momentum space of the cuprate lattice. Recall that in a periodic lattice the allowed momenta range from $k \in(-\pi / a, \pi / a)$, where $a$ is the lattice spacing. Thus $\Pi=(\pi, \pi)$ refers to the maximum allowed momentum of an electron on the cuprate fermi surface. $\Pi$ is distinct from, but not unrelated to, the operator $\pi_{i}$ defined in (B.1a)-(B.1c). The operator $\Delta$ has been defined previously in (B.1a)-(B.1c) and (B.2).

\section{Conflicts of Interest}

The author declares that there are no conflicts of interest regarding the publication of this paper.

\section{References}

[1] E. Demler and S.-C. Zhang, "Theory of the Resonant Neutron Scattering of High- $\mathrm{T}_{c}$ Superconductors," Physical Review Letters, vol. 75, p. 4126, 1995.

[2] E. Demler, W. Hanke, and S. Zhang, Reviews of Modern Physics, vol. 76, no. 3, pp. 909-974, 2004.

[3] S.-C. Zhang, "SO(5) Quantum Nonlinear sigma Model Theory of the High Tc Superconductivity," 1996, http://arxiv.org/abs/ cond-mat/9610140.

[4] S.-C. Zhang, "A Unified Theory Based on SO(5) Symmetry of Superconductivity and Antiferromagnetism," American Association for the Advancement of Science: Science, vol. 275, no. 5303, pp. 1089-1096, 1997.

[5] S. Murakami, N. Nagaosa, and M. Sigrist, "SO(5) Model of pwave Superconductivity and Ferromagnetism," Physical Review Letters, vol. 82, no. 14, pp. 2939-2942, 1999.

[6] L. Freidel and A. Starodubtsev, "Quantum gravity in terms of topological observables,” 2005, http://arxiv.org/abs/hep-th/ 0501191 .

[7] D. K. Wise, "MacDowell-Mansouri gravity and Cartan geometry," Classical and Quantum Gravity, vol. 27, no. 15, 2010.

[8] S. W. MacDowell and F. Mansouri, "Unified geometric theory of gravity and supergravity," Physical Review Letters, vol. 38, no. 14, pp. 739-742, 1977.

[9] K. S. Stelle and P. C. West, "Spontaneously broken de Sitter symmetry and the gravitational holonomy group," Physical Review D: Particles, Fields, Gravitation and Cosmology, vol. 21, no. 6, pp. 1466-1488, 1980.
[10] A. Randono, "Gauge Gravity: a forward-looking introduction," 2010, http://arxiv.org/abs/1010.5822.

[11] A. Randono, "Gravity from a fermionic condensate of a gauge theory," Classical and Quantum Gravity, vol. 27, no. 21, 2010.

[12] H. F. Westman and T. G. Zlosnik, "Exploring Cartan gravity with dynamical symmetry breaking," Classical and Quantum Gravity, vol. 31, no. 9, 2014.

[13] H. F. Westman and T. G. Zlosnik, "An introduction to the physics of Cartan gravity," Annals of Physics, vol. 361, pp. 330376,2015

[14] J. Magueijo, M. Rodríguez-Vázquez, H. Westman, and T. Złośnik, "Cosmological signature change in Cartan gravity with dynamical symmetry breaking," Physical Review D: Particles, Fields, Gravitation and Cosmology, vol. 89, no. 6, 2014.

[15] J. Ambjørn, A. Görlich, J. Jurkiewicz, and R. Loll, "Quantum Gravity via Causal Dynamical Triangulations," in Handbook of Spacetime, Springer, 2013.

[16] M. H. Ansari and F. Markopoulou, "A statistical formalism of Causal Dynamical Triangulations," Nuclear Physics B, vol. 726, no. 3, pp. 494-509, 2005, http://arxiv.org/abs/hep-th/0505165.

[17] M. H. Ansari, The Statistical Fingerprints of Quantum Gravity [Ph.D. thesis], University of Waterloo, 2008, http://uwspace .uwaterloo.ca/handle/10012/4074.

[18] B. Dittrich and J. Hnybida, "Ising Model from Intertwiners," 2013, http://arxiv.org/abs/1312.5646.

[19] A. Feller and E. R. Livine, "Ising spin network states for loop quantum gravity: a toy model for phase transitions," Classical and Quantum Gravity, vol. 33, no. 6, p. 065005, 2016.

[20] A. Feller and E. R. Livine, "Quantum surface and intertwiner dynamics in loop quantum gravity," Classical and Quantum Gravity, vol. 95, Article ID 124038, 2017.

[21] J. Wang, "Black hole as topological insulator," 2017, http://arxiv .org/abs/1703.09365.

[22] D. Kastor, S. Ray, and J. Traschen, "Enthalpy and the mechanics of AdS black holes," Classical and Quantum Gravity, vol. 26, no. 19, Article ID 195011, 16 pages, 2009.

[23] D. Kubiznak and R. B. Mann, "P - V criticality of charged AdS black holes," Journal of High Energy Physics, vol. 2012, article 33, 2012.

[24] D. Kubizňák and R. B. Mann, "Black hole chemistry," Canadian Journal of Physics, vol. 93, no. 9, pp. 999-1002, 2015.

[25] D. Kubiznak, R. B. Mann, and M. Teo, "Black hole chemistry: thermodynamics with lambda," Classical and Quantum Gravity, vol. 34, no. 6, Article ID 063001, 2017.

[26] Wikipedia, "High-temperature superconductivity - wikipedia, the free encyclopedia," 2017, https://en.wikipedia.org/w/ index.php?title=High-temperature_superconductivity_oldid= 812074078.

[27] S. Chakravarty, B. I. Halperin, and D. R. Nelson, "Twodimensional quantum Heisenberg antiferromagnet at low temperatures," Physical Review B: Condensed Matter and Materials Physics, vol. 39, no. 4, pp. 2344-2371, 1989.

[28] D. Vaid and S. Bilson-Thompson, Loop Quantum Gravity for the Bewildered: The Self-Dual Approach Revisited, Springer, 2017.

[29] C. P. Burgess and C. A. Lutken, "On the SO(5) Effective Field Theory of High $\mathrm{T}_{c}$ Superconductors," 1996, http://arxiv.org/abs/ cond-mat/9611070.

[30] C. P. Burgess and C. A. Lütken, " SO(5) invariance and effective field theory for high- ", Physical Review B: Condensed Matter and Materials Physics, vol. 57, no. 14, pp. 8642-8655, 1998. 
[31] S. Rabello, H. Kohno, E. Demler, and S. Zhang, "Microscopic Electron Models with Exact SO(5) Symmetry," Physical Review Letters, vol. 80, no. 16, pp. 3586-3589, 1998.

[32] D. Vaid, "Quantum Hall Effect and Black Hole Entropy in Loop Quantum Gravity," 2012, http://arxiv.org/abs/1208.3335.

[33] D. Vaid, "Non-abelian Gauge Fields from Defects in SpinNetworks," 2013, http://arxiv.org/abs/1309.0652.

[34] S. A. Hartnoll, C. P. Herzog, and G. T. Horowitz, "Building a Holographic Superconductor," Physical Review Letters, vol. 101, Article ID 031601, 2008.

[35] S. A. Hartnoll, C. P. Herzog, and G. T. Horowitz, "Holographic superconductors," Journal of High Energy Physics, vol. 2008, article 12, 2008.

[36] S. A. Hartnoll, "Lectures on holographic methods for condensed matter physics," 2010, http://dx.doi.org/10.1088/02649381/26/22/224002.

[37] C. P. Herzog, "Lectures on holographic superfluidity and superconductivity," 2009, http://dx.doi.org/10.1088/1751-8113/42/34/ 343001.

[38] M. Natsuume, "AdS/CFT Duality User Guide," 2014, http://arxiv .org/abs/1409.3575. 

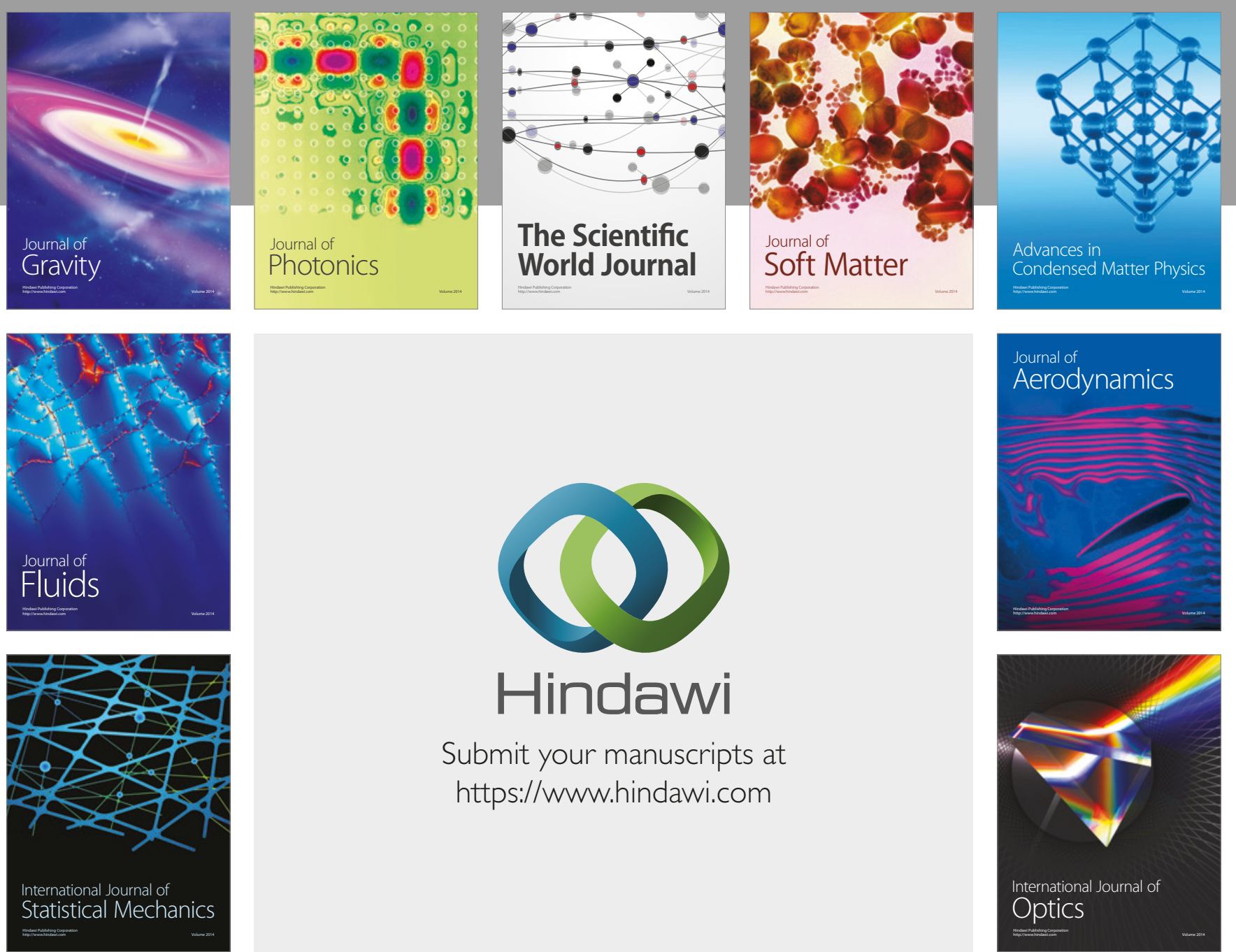

Submit your manuscripts at

https://www.hindawi.com
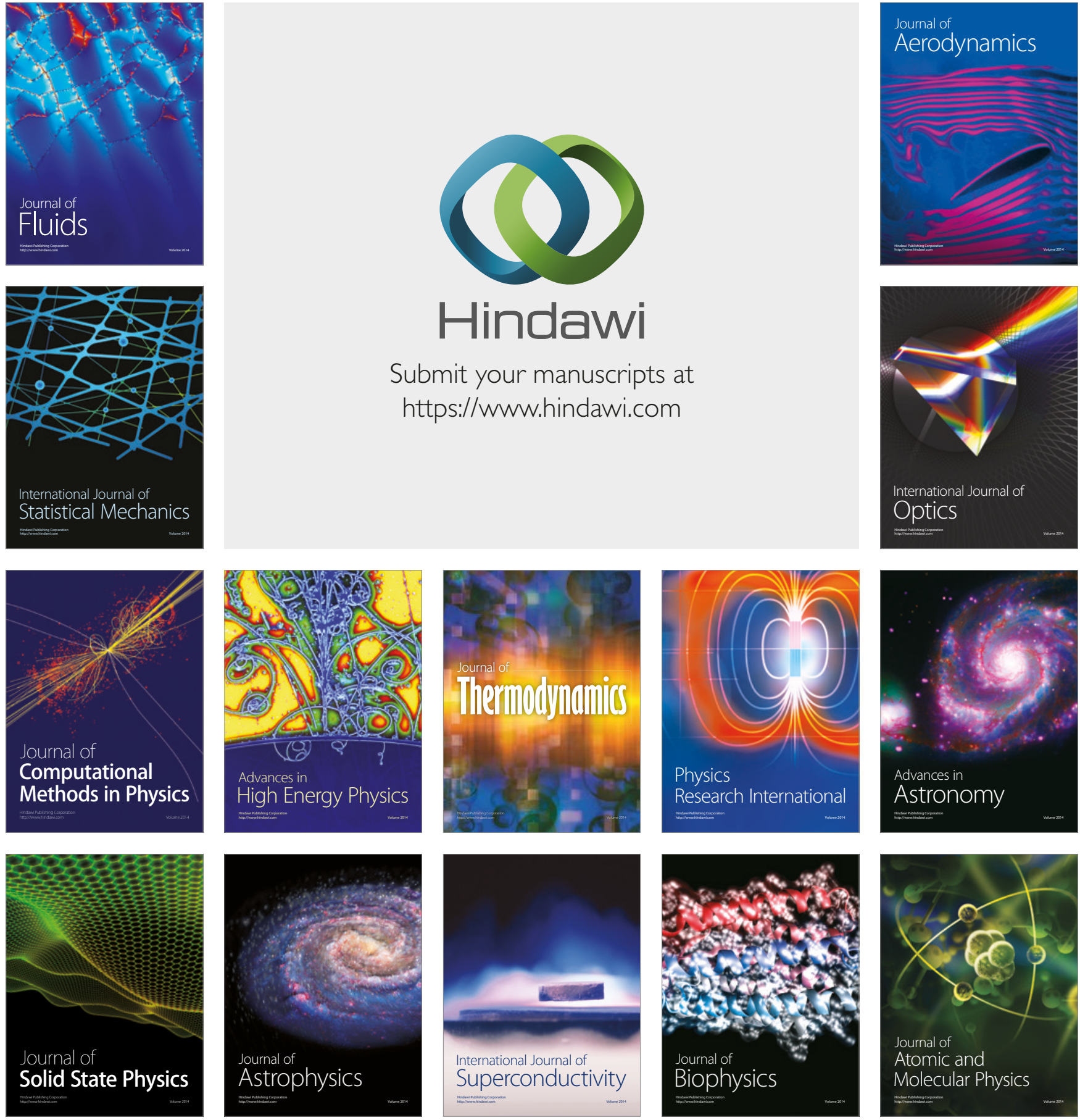